\title{
La terapia cognitivo conductual por internet no es inferior a la realizada en forma presencial para tratar el trastorno de ansiedad por enfermedad
}

\author{
Internet-delivered cognitive behavioral therapy is noninferior to a face-to-face modality for treating health anxiety
}

\section{Comentado de:}

Axelsson E, et al. JAMA Psychiatry. 2020;77(9):915-924. PMID: $32401286^{1}$

\section{Introducción}

El trastorno de ansiedad por enfermedad (a veces denominado hipocondriasis o ansiedad por la salud) es un problema de salud mental frecuente y a menudo crónico; se asocia con angustia, costos sustanciales y uso frecuente del sistema de atención médica. La terapia cognitivo-conductual (TCC) presencial es el tratamiento estándar, pero la accesibilidad de este tratamiento es limitada.

\section{Objetivo}

Probar la hipótesis de que la TCC suministrada por internet no es inferior a la TCC realizada en forma presencial en el tratamiento del trastorno de ansiedad por enfermedad.

\section{Diseño, lugar y pacientes}

Ensayo clínico aleatorizado de no inferioridad con análisis económico en salud desde la perspectiva de la sociedad. Fue realizado en una clínica de atención primaria en Suecia e incluyó pacientes con diagnóstico principal de trastorno de ansiedad por enfermedad que fueron auto-referidos o derivados de la atención de rutina. El reclutamiento comenzó en diciembre de 2014 y el último tratamiento finalizó en julio de 2017. Los datos de seguimiento se recopilaron hasta 12 meses después del tratamiento.

\section{Intervención}

Los pacientes fueron asignados al azar (1:1) a 12 semanas de TCC suministrada por internet o a TCC individual en forma presencial.

\section{Medición de resultados principales}

Se midió el cambio en los síntomas de ansiedad desde el inicio del ensayo hasta la semana 12, a los 6 y 12 meses. El análisis se realizó por intención de tratar y por protocolo (sólo con quienes completaron el tratamiento), utilizando el margen de no inferioridad de 2,25 puntos en el cuestionario Health Anxiety Inventory, el cual tiene un rango teórico de 0 a 54 .

\section{Resultados}

Del total de 204 pacientes (edad media: 39 años, desvío estándar: $12 ; 70 \%$ fueron mujeres), 102 (50\%) fueron asignados al azar a TCC administrada por internet, y 102 (50\%) fueron asignados a TCC presencial. Los límites superiores del Intervalo de Confianza del $95 \%$ (IC 95\%) unilateral para la diferencia en el cambio del desenlace principal estuvieron dentro del margen de no inferioridad (verTabla 1).
Tabla 1. Cambios en los síntomas de ansiedad documentados mediante la escala Health Anxiety Inventory desde el inicio del tratamiento, estimados en el análisis por intención de tratar. Se expresa en diferencia de medias entre los grupos de terapia cognitivo conductual por internet y en forma presencial.

\begin{tabular}{|c|c|c|}
\hline $\begin{array}{l}\text { Tiempo de segui- } \\
\text { miento }\end{array}$ & $\begin{array}{l}\text { Diferencia no } \\
\text { estandarizada, B } \\
\text { (IC } 95 \% \text { ) }\end{array}$ & $\begin{array}{l}\text { Diferencia } \\
\text { estandarizada, Cohen d } \\
\text { (IC 95\%)* }\end{array}$ \\
\hline Post tratamiento & $0,0(2,0)^{\star *}$ & $0,00(0,23)^{\star \star}$ \\
\hline A los 6 meses & $1,1(-1,1$ a 3,2$)$ & $0,12(-0,13$ a 0,37$)$ \\
\hline A los 12 meses & $2,4(-0,4$ a 5,1$)$ & $0,26(-0,05$ a 0,58$)$ \\
\hline
\end{tabular}

*Tamaño del efecto de Cohen d: se refiere a una medida estandarizada de efecto, que se calcula como la diferencia de medias dividida por la desviación estándar del punto final observado agrupado.

${ }^{* \star}$ Este intervalo de confianza es unilateral, de acuerdo con el análisis de potencia y plan de análisis de datos.

IC: Intervalo de confianza

Los terapeutas pasaron 10 minutos por paciente por semana en el tratamiento en línea frente a 45,6 minutos para la TCC presencial. En el análisis económico, el costo social neto fue menor en el tratamiento por internet (diferencia por punto del período de tratamiento: \$3.854). No hubo diferencias significativas entre los grupos en el número de eventos adversos, y no se informaron eventos adversos graves.

\section{Conclusiones}

La TCC suministrada por internet parece ser no inferior a la realizada en forma presencial en el tratamiento del trastorno de ansiedad por enfermedad, al tiempo que incurre en costos sociales netos más bajos y tiene el potencial de aumentar el acceso al tratamiento.

Fuente de financiamiento/Conflicto de interés de los autores: Los Dres. Axelsson y E. Hedman-Lagerlöf informaron haber recibido subvenciones de Instituto Karolinska y Consejo del Condado de Estocolmo (ALF Medicine) durante la realización del estudio.

Los Dres. Axelsson, Andersson, Ljótsson y E. Hedman-Lagerlöf son coautores de un libro de autoayuda para ansiedad por enfermedad grave, publicado entre el inicio de la investigación y la redacción del manuscrito.

Los doctores Ljótsson y E. Hedman-Lagerlöf son accionistas de DahliaQomit Inc, una compañía especializada en asesoramiento de síntomas psiquiátricos en línea; y de Hedman-Lagerlöf och Ljótsson psykologi $A B$ una empresa que crea y comercializa manuales de terapia cognitivo conductual en internet.

\section{Comentario}

En atención primaria las consultas por síntomas médicos inexplicables son muy comunes; hasta en $50 \%$ de los casos no se encuentra una causa somática, y muchos de estos pacientes se volverán consultadores crónicos ${ }^{2}$. Dentro de las patologías que padece esta población se encuentra el trastorno de ansiedad por enfermedad, definido por DSM- $5^{3}$ como un paciente que está preocupado por tener o adquirir un trastorno grave, tiene mínimos síntomas, está muy preocupado por la salud, se 
alarma con facilidad frente a problemas de salud personales, comprueba repetidamente el estado de salud o evita las citas médicas, se ha preocupado por la enfermedad durante un tiempo mayor o igual a 6 meses y sus síntomas no se atribuyen a otro trastorno mental.

La prevalencia de esta patología es mayor entre mujeres de 35 a 45 años de edad. El tratamiento de primera línea es la TCC, seguida por otras psicoterapias, y puede considerarse como tercera línea el uso de medicación antidepresiva ${ }^{4-6}$.

Si bien Argentina es el país con más psicólogos por habitante del mundo ${ }^{7}$, el acceso a la TCC es escaso ya que más del $80 \%$ de los profesionales se dedican al psicoanálisis.

Existen estudios previos que evalúan y corroboran la efectividad de la TCC por internet ${ }^{8}$, pero éste es el primero que permite afirmar que esta modalidad no es inferior a la TCC administrada en forma presencial, requiriendo menos tiempo, generando menos costos en salud y mejorando de esta manera la accesibilidad al tratamiento.

Si bien el estudio demuestra la no inferioridad en los resultados a corto plazo (hasta las 12 semanas), los resultados sugieren que la eficacia a largo plazo (12 meses) sería algo mayor en la TCC presencial, por lo que las estimaciones de costo-utilidad deben ser interpretadas con cautela.

\section{Conclusiones del comentador}

La dificultad en el acceso a la TCC, sumada a la situación epidemiológica actual por la pandemia de COVID-19, nos obliga a centrar las intervenciones médicas en el área de la telemedicina y las consultas virtuales. La TCC administrada por internet podría ser considerada una primera línea de tratamiento para pacientes con trastorno de ansiedad por enfermedad en estas circunstancias.

Alan Gauna [ Servicio de Medicina Familiar y Comunitaria, Hospital Italiano de Buenos Aires. alan.gauna@hospitalitaliano.org.ar ]

Gauna A. La terapia cognitivo conductual por internet no es inferior a la realizada en forma presencial para tratar el trastorno de ansiedad por enfermedad . Evid Actual Pract Ambul. 2020;4(23):e0002082. Comentado de: Axelsson E, et al. Effect of Internet vs Face-to-Face Cognitive Behavior Therapy for Health Anxiety: A Randomized Noninferiority Clinical Trial. JAMA Psychiatry. 2020;77(9):915-924. PMID: 32401286

\section{Referencias}

1. Axelsson E, Andersson E, Ljótsson B, et al. Effect of Internet vs Face-to-Face Cognitive Behavior Therapy for Health Anxiety. JAMA Psychiatry. 2020;77(9):915-915. Available from: 10.1001/jamapsychiatry.2020.0940;https://dx.doi.org/10.1001/jamapsychiatry.2020.0940.

2. Verhaak PF, Meijer S, Visser A, et al. Persistent presentation of medically unexplained symptoms in general practice. Family Practice. 2006;23(4):414-420. Available from: 10.1093/fampra/cml016;https://dx.doi.org/10.1093/fampra/cml016.

3. Asociación Americana De Psiquiatría. DSM-5. Manual Diagnóstico y Estadístico de los Trastornos Mentales. Editorial Médica Panamericana; 2018.

4. Thomson AB, Page LA. Psychotherapies for hypochondriasis. Cochrane Database Syst Rev. 2007;17(4):CD006520. Available from: 10.1002/ 14651858.CD006520.pub2.

5. Greeven A, van Balkom AJLM, Visser S, et al. Cognitive Behavior Therapy and Paroxetine in the Treatment of Hypochondriasis: A Randomized Controlled Trial. Am J Psychiatry. 2007;164(1):91-99. Available from: 10.1176/ajp.2007.164.1.91;https://dx.doi.org/10.1176/ajp.2007.164.1.91

6. Williams C, House A. Cognitive behaviour therapy for health anxiety. Lancet . 2014;383(9913):190-191. Available from: 10.1016/s0140-6736(13) 62107-8.

7. Mental Health ATLAS 2017. In: World Health Organization (WHO). Geneva; 2018.Available from: https://apps.who.int/iris/bitstream/handle/10665/ 272735/9789241514019-eng.pdf?ua=1.

8. Hedman E, Andersson G, Andersson E, et al. Internet-based cognitive-behavioural therapy for severe health anxiety: randomised controlled trial. Br J Psychiatry. 2011;198(3):230-236. Available from: 10.1192/bjp.bp.110.086843. 